\title{
ALZHEIMER'S DISEASE: A CONSEQUENCE OF IMPAIRED MITOPHAGY?
}

\author{
VIVEK SHARMA* \\ Department of Pharmacology, Government College of Pharmacy, Rohru, Shimla - 171 207, Himachal Pradesh, India. \\ Email: viveksharma_pharma@yahoo.co.in
}

Received: 10 July 2018, Revised and Accepted: 01 November 2018

ABSTRACT

Neurodegenerative disorders involve complexities of the pathologies, characterized by the progressive loss of neuronal viability, leading to severe physical and cognitive impairments in affected patients. These disorders although may differ in clinical outcomes yet they share common features such as aggregation of neurotoxic metabolites and perturbed cellular and neuronal homeostasis. Mitochondrion is an indispensable organelle for neuronal survival, and its role and place have become critical in research arena of aging and neurodegenaration. The accumulation of damaged mitochondria has been linked to normal aging and multiple age-related disorders including Alzheimer's disease (AD). Survival and proper function of mitochondria depend on several attributes that include mitochondrial biogenesis and fusion and fission. Mitophagy is an utmost requirement for degradation and removal of damaged mitochondria where the target mitochondria are identified by the autophagosomes and delivered to the lysosome for degradation. Mitophagy plays important roles in mitochondrial homeostasis, neuroprotection, and resistance to neurodegeneration. AD besides other characteristic features involves mitochondrial dysfunctional, bioenergetic deficit, and altered mitophagy. The autophagy/lysosome pathway that removes damaged mitochondria (mitophagy) is compromised in AD, resulting in the accumulation of dysfunctional mitochondria that contribute to synaptic dysfunction and cognitive deficits by triggering $A \beta$ and Tau accumulation through increases in oxidative damage and cellular energy deficits. The present work reviews the various implications of mitophagy in relevance to the pathology of AD.

Keywords: Alzheimer's disease, Cognitive deficits, Energy failure, Mitochondrion, Mitophagy.

(C) 2019 The Authors. Published by Innovare Academic Sciences Pvt Ltd. This is an open access article under the CC BY license (http://creativecommons. org/licenses/by/4. 0/) DOI: http://dx.doi.org/10.22159/ajpcr.2019.v12i2.28407

\section{INTRODUCTION}

The expansion of the mitochondrial research is attributable to the convergence of key signaling pathways and biological processes onto the mitochondrion and their relevance to different pathologies [1]. As life evolved from unicellular organisms over the past 1.2-1.5 billion years and mitochondria played a permissive role in the evolution of multicellular organisms [2], mitochondria are intimately linked to a number of basic cellular and physiological functions [3].

The origin of mitochondria in the eukaryotic cell is traced back to the merger of two cells. Billions of years ago, the mitochondrion developed from an $\alpha$-proteobacterium that was taken up by a host cell providing an evolutionary advantage which kept it alive. Different theories exist to whether the host cell was of eukaryotic origin or a prokaryotic and later theory being favored. Over time, most of the bacterial genome was transferred to the genome of the host, but a small percentage remained in the mitochondrion, and thus, mitochondrial machinery is encoded by two genomes in separate compartments within the same cell, the mitochondrial DNA (mtDNA) and the nuclear DNA (nDNA) [2,4].

The mitochondrion is formed by two phospholipid bilayers enclosing the mitochondrial matrix. The outer mitochondrial membrane faces the cytosol and is permeable to metabolites required for mitochondrial function. The inner mitochondrial membrane, in contrast, tightly regulates the transport of even the smallest ions, the smallest being the hydrogen ion which eventually drives phosphorylation of adenosine diphosphate (ADP) to adenosine triphosphate (ATP). The inner mitochondrial membrane is highly folded to further increase its surface area which forms cristae, the regions at which mitochondrial ATP is generated.

The mitochondrion is highly versatile, dynamic and has multiple functions. It can buffer calcium and, in doing so, maintains cytosolic and mitochondrial calcium levels to regulate the diverse set of cellular enzymatic reactions. It also regulates metabolite levels to provide precursors for the synthesis of amino acids, nucleotides, and fatty acids and is involved in the cellular response to stress [5]. Mitochondria are also crucial in apoptosis, necrosis, autophagy, stress regulation, production of lipids and carbohydrates, $\mathrm{Ca}^{2+}$ storage, and innate immunity [6].

Diseases specifically linked to mitochondrial dysfunction vary from the well known (glaucoma, inflammation, neurodegenerative diseases (NDs), type 2 diabetes, cancers, especially those involving prostate and colon, cardiomyopathies, and dysrhythmias) to the less well known (Friedreich's ataxia) to a group of relatively obscure disease states (Kearns-Sayre syndrome, Leber's hereditary optic neuropathy, mitochondrial encephalopathy, lactic acidosis, and strokes, myoclonic epilepsy with ragged red fibers, and mitochondrial neurogastrointestinal encephalomyopathy [7]. Deficiencies in energy metabolism and bioenergetic failure are characteristic of mitochondrial and epigenomic disease states [8] and have been implicated in a variety of human disease states, especially in organs where there is a high level of energy consumption, for example, the brain. The brain, with only $2 \%$ of total body weight, represents $20 \%$ of the total oxygen consumption in the body.

\section{Mitochondria and energy production}

The primary function of the mitochondrion is to produce energy which it produces in the form of ATP through a process referred to as oxidative phosphorylation (OXPHOS). The energy stored in carbohydrates, fats, and proteins is broken down to generate high-energy electrons which are carried down a chain of protein complexes at the inner mitochondrial membrane, the respiratory chain. This electron transport is coupled to proton pumping across the inner mitochondrial membrane, generating a proton gradient which is used by the ATP synthase, also known as complex V (CV), to phosphorylate ADP $[9,10]$.

Human mtDNA is approximately 16.6 base pairs long, forming a closed, double-stranded structure [11]. Each mitochondrion contains 
between 2 - 10 mtDNA copies that consist of 37 genes which encodes 22 transfer, 2 ribosomal DNAs and 13 proteins. The proteins thus encoded include the enzymes which are involved in the OXPHOS pathway for ATP production. OXPHOS units are coded by both nDNA and mtDNA, with the former contributing somewhere in excess of 1000 proteins that are essential for mitochondrial function [8,12]. Of these, 705 are under the transcriptional control of estrogen-related receptors that are responsible for the integrated control of mitochondrial metabolism [12].

The OXPHOS pathway consists of five different electron transport chain (ETC) complexes located on the inner mitochondrial membrane that together contributes to the generation of the mitochondrial electrochemical gradient. These complexes are composed of proteins that originate from both nDNA and mtDNA $[8,13]$. Complex I consists of 45 peptide subunits, 7 originating from mtDNA with the remainder from nDNA. Complex II has four subunits, all of which are derived from nDNA, and Complex III has 11 subunits, only one of which originates from mtDNA. Complex IV has 12 subunits, 3 of which are derived from mtDNA, and complex IV has approximately 16 subunits, 2 of which are from mtDNA. The fifth ETC complex, CV, is ATP synthase [14].

Approximately $90 \%$ of ATP arises from mitochondria. In complex I ( $\beta$-nicotinamide adenine dinucleotide (NADH) dehydrogenase), two electrons are removed from NADH and transferred to the lipid-soluble carrier, ubiquinone $(\mathrm{Q})$ forming the reduced product, ubiquinol $(\mathrm{QH} 2)$ that can freely diffuse in the membrane. Complex I thus leads to the translocation of four protons across the membrane to produce a proton gradient.

In complex II (succinate dehydrogenase), additional electrons are delivered from succinate through flavin adenine dinucleotide (FAD) to the quinone pool $(\mathrm{Q})$ and transferred through FAD to Q. In complex III (QH-cytochrome c [CytC] reductase), six electrons are removed from $\mathrm{QH} 2$, two of which are sequentially transferred to two molecules of CytC, a water-soluble electron carrier located in the intermembrane space, and four to the Qi site where the quinone moiety in ubiquinone is reduced to quinol contributing to the proton gradient. In complex IV (CytC oxidase), four electrons contributed by four CytC molecules are transferred to molecular oxygen $\left(\mathrm{O}_{2}\right)$, resulting in two molecules of water. Concomitantly, four protons translocate across the membrane, adding further to the proton gradient. The latter is then used in $\mathrm{CV}$, the F 0/F 1 ATP synthase complex to produce ATP through OXPHOS [15].

Mutation rates in mtDNA are generally 2-3-fold higher than those occurring in nDNA due to consequence of the proximity of mtDNA to the reactive oxygen species (ROS) produced by electron leakage from complexes I and III of the ETC. The scenario is further complicated with inefficient DNA repair mechanisms and a lack of protective histones on mtDNA which are thought to affect mitochondrial protein synthesis, protein-encoding genes and mRNA, and ultimately mitochondrial function. These are complemented by rearrangements, deletions, and insertions in mtDNA and their altered interactions with nDNA, the latter reflecting defects in mitochondrial transport processes [13]. Thus, the age-related accumulation of somatic mtDNA mutations that can lead in time to decreased mitochondrial function has been associated with an increased rate of aging $[8,16,17]$. A variety of conditions (hypoxia, stress, trauma, blood glucose levels, aberrant circadian rhythms, etc.) and agents/mechanisms (phosphorylation, DNA methylation/acetylation, Akt/protein kinase B signaling, calcium homeostasis, estrogen-related receptor signaling, heat shock proteins, soluble adenylyl cyclase, receptorinteracting protein 3 kinase, target of rapamycin kinases, peroxisome proliferator-activated receptor- $\alpha$, adenosine monophosphateactivated protein kinase, $\beta$-amyloid, sirtuin-1, etc.) are involved in both modulating transcription of the mitochondrial genome and the function of the transcribed proteins. Mutated proteins such as huntingtin in HD; amyloid $(A \beta)$ in Alzheimer's disease (AD); superoxide dismutase (SOD) 1 in amyotrophic lateral sclerosis; and parkin, DJ1, and $\alpha$-synuclein in Parkinson's disease have been localized to mitochondrial membranes [18] where they can alter ETC function to increase ROS production [14]

\section{MITOCHONDRIAL DYNAMICS}

Aging, at the cell level, is an increasingly incapacity to recycle organelles and macromolecules. High-energy requirement tissues such as the brain are highly dependent on mitochondria. In a single neuron, hundreds to thousands of mitochondria are contained [19].

Mitochondria form networks throughout the cell through the opposing processes of fission and fusion [20]. The latter is critical to the maintenance of mitochondrial function because it affects the repair of dysfunctional and damaged mitochondria in addition to intermixing DNA and proteins between mitochondria [21]. Fusion involves the merging of the inner and outer membranes from two mitochondria to facilitate the GTPase-dependent exchange of materials to aid in mitochondrial repair. Fission occurs when a mitochondrion splits in two. When this process occurs in the presence of decreased fusion, it can lead to a fragmented mitochondrial phenotype that is widespread in both necrosis and apoptosis. Deficient fission and fusion mechanisms are, thus, key events in mitochondrial disease causality [14].

In aging, proteasome activity decreases, autophagy is impaired, and mitochondrial dysfunction emerges with a decrease of OXPHOS, betaoxidation, and Krebs cycle as well as an increase of oxidative stress that, in turn, damages mitochondria.

As the age progresses, vulnerability of mitochondria also increases. Aging is tightly linked to mtDNA deletions, point mutations, and ROS. In addition, mtDNA deletions and point mutations accumulate over time. This leads to energetics impairment, increased ROS production, mtDNA lesions, and the decline of mitochondrial respiration. ROS targets mtDNA since there are no histones and efficacious proofreading systems and this brings more point mutations and deletions. In the mitochondria, deletions expand clonally over time and their number correlates linearly with the cell age [22].

\section{ROS and brain}

OXPHOS occurring in the mitochondria is a major source of ATP, but the by-product of this process is free radicals or ROS, reactive nitrogen species (RNS), and carbon- and sulfur-centered radicals [23]. Low amounts of ROS are considered essential for neuronal development and function, as ROS-generated nitrous oxide and carbon monoxide promote important physiologic functions, such as long-term potentiation (LTP) via glutamate-dependent mechanisms [24,25]. Under normal conditions, deleterious effects of ROS production during aerobic metabolism are neutralized by the antioxidant system, and in this manner, the brain effectively regulates its oxygen consumption and redox generation capacity. When ROS production exceeds the scavenging capacity of antioxidant response system, extensive protein oxidation and lipid peroxidation occur, causing oxidative damage, cellular degeneration, and even functional decline. For example, high ROS concentrations reportedly diminish LTP and synaptic signaling and brain plasticity mechanisms [24-26].

The brain, with its rich lipid content, high-energy demand, and weak antioxidant capacity, becomes an easy target of excessive oxidative insult. Phospholipids in the brain are particularly vulnerable entities for ROS-mediated peroxidation, but proteins and DNA also are targeted by ROS, which becomes particularly problematic during aging, as aged brains have been reported to exhibit high levels of oxidative stressinduced mutations in the mtDNA [27-29]. Therefore, ROS accumulation is a cellular threat that, if it exceeds or bypasses counteracting mechanisms, can cause significant neuronal damage [29].

Two kinds of protective mechanisms operate in the brain to tackle the threat posed by ROS, the antioxidant enzyme system and the lowmolecular-weight antioxidants [30,31]. The antioxidant enzyme system includes SOD, glyoxalase, glutathione reductase, glutathione peroxidase (GPX), and catalase (CAT). SOD enzymes, including Cu-Zn SOD and Mn-SOD, facilitate spontaneous dismutation of superoxide radicals to generate $\mathrm{H}_{2} \mathrm{O}_{2}$, which is further removed by CAT and GPx enzymes [32]. 
The low-molecular-weight antioxidants include glutathione, uric acid, ascorbic acid, and melatonin, which offer neutralizing functions by causing chelation of transition metals [33].

In response to oxidative and nitrosative stress, cells increase their antioxidant defenses through activation of nuclear factor erythroid 2-related factor (Nrf2), an important transcription factor [34]. Nrf2 is a key component of this control system and recognizes the antioxidant response element found in the promoter regions of many genes that encode antioxidants and detoxification enzymes such as heme oxygenase 1 (HO-1), NAD(P)H dehydrogenase quinone 1, SOD1, GPx1, and CAT. Thus, Nrf2 pathway activation occurs to combat the accumulation of ROS and RNS species. Due to its protective properties, Nrf2 has been proposed as a pharmacological target in pathologies with neuroinflammatory and oxidative features, including neurodegenerative and neuropsychiatric diseases. When activated, Nrf2 increases the expression of several endogenous antioxidants. Moreover, on persistent inflammation and increased ROS levels, as observed during several psychiatric episodes, tissue antioxidant defense mechanisms are saturated to the point they become ineffective $[26,34]$.

Mechanisms by which ROS causes cerebral tissue damage are not well understood, but ROS is reported to trigger a variety of molecular cascades that increase blood-brain barrier permeability and alter brain morphology, thus causing neuroinflammation and neuronal death [35]. Involvement of hypothalamic-pituitary-adrenal axis-mediated glucocorticoid receptor signaling, glutamate toxicity, and N-methyl-Daspartate receptor signaling systems also has been suggested [36,37].

Biochemically, it is evident that different neurons have different levels of vulnerability to oxidative stress. For example, hippocampus, amygdala, and cerebellar granule cells have been reported as the most susceptible to oxidative stress in some studies [38], and cognitive deficits are attributed to these regions $[39,40]$. Hippocampus seems to be at the hot seat, and it appears that this brain region undergoes major biochemical changes that ultimately determine neuronal connections and function. Within the hippocampus, it is well known that the dentate gyrus (DG)-cornu ammonis (CA) 3 system exhibits structural plasticity with regenerative/remodeling capacity [41]. Furthermore, several studies have suggested that pyramidal cells of CA3 and granule cells of the DG are oxidative stress-prone areas, whereas others have suggested that pyramidal cells of CA1 are more susceptible to oxidative damage $[42,43]$.

\section{AUTOPHAGY}

Autophagy is an evolutionarily conserved process, in which cytoplasmic substrates are engulfed in an autophagic vesicle, fused to lysosomes, and degraded. Autophagy is a self-elimination process through which cells degrade their own components while recycling amino acids and other building parts that can eventually be reused.

Autophagy is a cellular degradation process in which cytosolic cargos are degraded by lysosomes and it can be divided into three subtypes - microautophagy, chaperone-mediated autophagy, and macroautophagy. In microautophagy, cytosolic cargo is directly engulfed by the lysosome through invagination of the lysosomal membrane, whereas in chaperone-mediated autophagy, cargo is selectively recognized by cytosolic chaperones that deliver the cargo to a translocation complex on the lysosomal membrane. Macroautophagy (autophagy) involves the formation of the autophagosome, a doublemembraned organelle that forms around cytosolic cargo and subsequently degrades the cargo by lysosomal fusion [44].

Like other cells, neurons too accumulate protein aggregates and damaged organelles such as mitochondria that must be degraded by autophagy to maintain cellular homeostasis. However, neurons are postmitotic cells that are highly polarized with both dendritic and axonal compartments, which can extend over distances many times greater than their cell soma. Consequently, neurons require motor proteins to actively supply proteins and organelles to these distal processes and to drive the transport of signaling molecules and degradative compartments back to the cell body. Neurons also have high energetic demands due to their elaborate morphologies and their need to maintain active electrochemical signaling. They require efficient recycling of proteins and organelles at the synapse, as well as throughout the axon, dendrites, and cell soma, making autophagic degradation particularly crucial in neurons. Autophagy is also required for neuronal development and the maintenance of axonal homeostasis $[45,46]$.

The defective autophagy induces protein aggregation and neurodegeneration $[46,47]$. Although autophagy is efficient in younger neurons, autophagy proteins such as beclin-1, Atg5, and Atg7 decline with age [48] potentially contributing to the late onset of many NDs [49].

\section{Mitophagy}

Neurons are highly dependent on mitochondrial metabolism for their high-energy demands. As such, the mitochondrial network needs to be in perfect working order. Moreover, the unique morphology of neurons, with their axons and dendrites, means that mitochondria must be recruited to distant parts of the cell through axonal transport to satisfy energy demands at those sites. Consequently, neurons are vulnerable to defects in axonal trafficking. Finally, due to the postmitotic state of neurons, cellular division cannot act as a diluting factor for components accumulating within the cell: This means that neurons require an adequate "cleaning system" to eliminate any damaged or unwanted mitochondria that could be a source of ROS or an inducer of programmed cell death. Given their essential role in neuronal viability, any alterations in mitochondrial function could lead to neuronal death, thus explaining why mitochondrial dysfunction has been linked to numerous neurodegenerative disorders [50].

When mitochondria become "old" or damaged and are no longer able to efficiently produce energy, they are eliminated from the cells by a process known as mitophagy, where autophagosomes engulf and remove them from the internal cell environment, and they are then degraded by lysosomes [51].

Mitophagy regulates the number of mitochondria according to metabolic demands, and it also serves as a quality control mechanism to eliminate damaged mitochondria. Mitophagy is preceded by mitochondrial fission, which divides mitochondria into pieces for engulfment, followed by disposal [52].

Mitophagy is the only known pathway through which whole mitochondria can be selectively eliminated. This mechanism was first characterized in yeast in 2004 [53]. Later, in 2006, the term "mitophagy" was used by LeMaster Group in relation to mammalian cells; they showed that depolarized mitochondria localize inside light chain 3 (LC3)-GFP-labeled autophagosomes before being completely degraded [54].

Mitophagy follows a general pattern involving a receptor-mediated mechanism, whereby the receptors physically connect the mitochondria to be eliminated with LC3-II, the main component of the autophagosomal membrane. This connection is established through the LC3-interacting region (LIR) present in all receptors [55]. The nature and origin of mitophagy receptors can vary depending on the type of mitophagy; some receptors are proteins or lipids localized in the mitochondrial membrane, while others are non-mitochondrial proteins that recognize and simultaneously bind ubiquitinated chains on the mitochondrial surface and LC3-II on the nascent autophagosome structure. These receptors contain both the LIR motif to bind LC3 and the ubiquitin-binding domain to bind ubiquitin (UB) chains on the targeted mitochondria [55].

Mitophagy is a part of the normal cellular turnover of mitochondria and is essential to keep cells healthy. If this process is impaired or fails, it leads to the accumulation of dysfunctional mitochondria, which induces oxidative stress within a cell, leading to cellular degeneration. The 
role of mitochondrial dysfunction and the impairment of mitophagy in disease and pathology is starting to be elucidated, highlighting the importance of finding therapeutic strategies that help promote mitochondrial clearance and quality control $[51,56]$

\section{Mechanistic view of mitophagy}

In mitophagy, the enzymes E3 ubiquitin ligases such as parkin (also known as PARK2) and the protein kinase PTEN-induced putative kinase 1 (PINK1) collaborate to ubiquitylate or "tag" mitochondrial membrane proteins of damaged mitochondria with ubiquitin, a $7.5 \mathrm{kDa}$ regulatory protein. This tagging promotes the clearance of these poorly functioning mitochondria from normal healthy cells. During the mitophagy process, the initiating event is the loss of the mitochondrial membrane potential of damaged mitochondria (and eventually the opening of mtPTPs), which leads to the accumulation of the PINK1 at the outer membrane of the mitochondrion, which in turn phosphorylates parkin, Mfn2, and other proteins. Parkin is a cytosolic E3-ubiquitin ligase that is selectively recruited to damage mitochondria. Activated parkin ubiquitinates the outer mitochondrial membrane proteins. Ubiquitin-tagged mitochondrial proteins, in turn, assist in the recruitment of autophagosomal membranes to mitochondria and trigger their fragmentation and degradation [57]. Impaired mitophagy may contribute to the decline of mitochondria function leading to the aging phenotype [58].

In addition to classical PINK1/parkin-related mitophagy, other mitophagy receptors have been discovered, including AMBRA1, FUNDC1, and Nix/BNIP3L in mammals. AMBRA1 can bind to LC3 and induce mitophagy in either a parkin-dependent or parkin-independent manner [59]. FUNDC1 is a mitochondrial outer membrane protein that can regulate mitochondrial fusion/fission (Drp1 and Opa1) to induce mitophagy in hypoxic conditions $[60,61]$

\section{MITOPHAGY AND AD}

Although the origin, progression, and heterogeneity of NDs differ, these disorders are characterized by common features at the molecular level. These include: (i) Accumulation of aggregated misfolded proteins; (ii) impairment of degradative processes including autophagy and the ubiquitin-proteasome system; (iii) oxidative stress; (iv) neuroinflammation; and (v) impaired mitochondrial function comprising mitochondrial dynamics, trafficking, and turnover [50].

$\mathrm{AD}$ is the most common form of dementia and is characterized by a progression from episodic memory problems to severe cognitive decline and complete dependence of the patient on caregivers. The disease-defining histopathological abnormalities, extracellular deposits of amyloid $\beta$-peptide $(\mathrm{A} \beta)$ and intraneuronal accumulation of hyperphosphorylated Tau (Tau) are the primary disease defining features occurring in the entorhinal cortex and hippocampus [62,63].

A widely documented abnormality in neuronal physiology that occurs before the onset of discernable cognitive deficits in those who develop $\mathrm{AD}$ is impaired glucose utilization and mitochondrial abnormalities in $\mathrm{AD}$, elucidating the underlying molecular mechanisms and cellular consequences of mitochondrial deficits. The mitochondrial cascade hypothesis states that each individual's genetically determined and environmentally influenced mitochondrial function are primary factors influencing late-onset AD pathology [64].

Increasing evidence suggests that inhibition of the clearance of damaged mitochondria, along with concurrent increases in oxidative stress levels, results in the accumulation of dysfunctional neurons in $\mathrm{AD}$. To be removed by mitophagy, the autophagosome containing the mitochondrion must fuse with a lysosome to form autolysosomes in which proteases degrade the mitochondrion. Neurons exhibiting an abnormal accumulation of autophagosomal vacuoles are a prominent feature in $\mathrm{AD}$, and their accumulation may result from lysosomal dysfunction (elevated $\mathrm{pH}$ ), perhaps secondary to dysregulation of neuronal $\mathrm{Ca}^{2+}$ homeostasis $[65,66]$. The undegraded dysfunctional mitochondria accumulate in the soma which may result from the combination of local lysosome dysfunction and impaired mitochondrial transport [67]. The impairment of lysosomal function in healthy cells results in neuronal phenotypes like those in $\mathrm{AD}$ [67].

Mitophagy- and mitochondrial dynamics-related proteins affected in AD include those involved in mitochondrial fission and fusion (Drp1 and mitofusin), mitochondrial biogenesis (PGC-1 $\alpha$ ), and mitochondrial responses to bioenergetic and oxidative challenges (SIRT3 and SIRT1) [68,69]. Mitochondrial fission and fusion are controlled by specialized proteins such as Fis1 and Drp1 [70,71] and Opa1, respectively [72]; the levels of each of these proteins determine the amount of fission and fusion occurring. Before fission, damaged DNA and proteins are segregated to one side of the mitochondrion such that, on fission, one of the "daughter" mitochondrion contains damaged molecules and is targeted for mitophagy, while the other daughter mitochondrion is now pristine [73]. Cell culture and animal models of $\mathrm{AD}$ have demonstrated excessive fission of mitochondria concomitant with an accumulation of dysfunctional mitochondria, possibly as the result of lysosomal dysfunction [74]. Some studies have demonstrated the increased levels of Drp1 in the brains of AD patients and mice [75].

Analyses of postmortem brain tissue from AD patients and age-matched control subjects have revealed reduced expression of genes related to mitochondrial biogenesis including PGC-1 $\alpha$, TFAM, and NRF2 [76]. Further, Beclin1, which is necessary for the initiation of autophagy, has been shown to be reduced in the brains of patients with $\mathrm{AD}$, and the reduction of beclin 1 leads to decreased autophagy, the accumulation of $\mathrm{A} \beta$ peptides, and neurodegeneration in mouse models of $\mathrm{AD}$ [77].

\section{Amyloid $\beta$ and mitophagy}

The formation of extracellular senile plaques containing $A \beta$ starts with the processing of amyloid precursor protein (APP) by $\beta$ - and $\gamma$-secretases; some of the resulting Ab peptides can spontaneously aggregate to form oligomers and subsequently insoluble fibrils in a process called amyloidogenesis. These oligomeric Ab's are the precursors of the insoluble amyloid fibrils and are considered responsible for the neurotoxicity present in AD. This amyloidogenic process can occur both externally at the plasma membrane, where APP localizes, and inside the cell, in the membranes of different cellular compartments where the APP along with the b- and g-secretases is also present. These intracellular compartments include endosomes, the trans-Golgi network, the endoplasmic reticulum, lysosomes, autophagosomes, multivesicular body, and mitochondria [78]. Thus, $A \beta$ can be found both extracellularly and intracellularly where it can interfere with different cellular pathways. The endosomal system participates at different levels in the production of $A \beta$. These include the processing of APP to A $\beta$ at the endosomal membrane, the internalization of external $A \beta$ by endocytosis, and the intracellular trafficking and secretion of $A \beta$ to the outside of the cell. Changes in the endosomal system have been observed in $\mathrm{AD}$, and several proteins regulating this pathway shown to be altered, thereby affecting the regulation, sorting, trafficking, and secretion of endosomal vesicles. As a result, levels of intracellular $A \beta$ increase and promote further endosomal dysfunction as a consequence [79].

In $\mathrm{AD}$, mitochondrial dysfunction promotes amyloid production through oxidative stress production. Oxidative stress increases $\gamma$-secretase activity by a mechanism involving covalent modification of the $\gamma$-secretase complex protein nicastrin by the membrane lipid peroxidation product 4-hydroxynonenal [80]. A major source of 4-hydrox-ynonenal is arachidonic acid, a lipid enriched in mitochondrial membranes. Together, accumulating evidence suggests that amyloidogenic APP processing in late-onset AD occurs due to an increased generation of ROS by dysfunctional mitochondria in neurons.

\section{Tau and mitophagy}

Microtubule-associated protein Tau is another predominant player in AD. Microtubules are a component of the cellular cytoskeleton on which mitochondria, lysosomes, and other organelles are transported. Tau 
binds and stabilizes microtubules, but when Tau is hyperphosphorylated it detaches from and thereby destabilizes microtubules resulting in their depolymerization. The accumulation of pTau aggregates and fibrils in the cell bodies and neurites of degenerating neurons is strongly correlated with cognitive deficits in AD [81].

Studies of experimental models have provided evidence that mitochondrial dysfunction can result in pTau, microtubule depolymerization, and neurofibrillary tangle-like pathology. AD- like pTau occurs in hippocampal neurons with chronically elevated $\mathrm{Ca}^{2+}$ levels due to exposure to glutamate, a condition in which mitochondrial function is impaired. By initiating membrane lipid peroxidation, ROS generated by mitochondria may also promote pTau and its aggregation [82]. Similarly, the deletion of AFG3L2, a protease that processes misfolded proteins in the inner mitochondrial membrane, leads to mitochondrial network fragmentation, defective anterograde mitochondrial transport, and subsequent Tau hyperphosphorylation in neurons [83]

Further, a basic cellular metabolite, NAD+ exists in all living cells including brain cells, where it plays fundamental roles in neuroplasticity and cellular stress resistance. In cell metabolism, NAD+ and its reduced form NADH are necessary for glycolysis, tricarboxylic acid cycle, OXPHOS, and ATP production. As neurons consume relatively large amounts of energy, they are extremely sensitive to decreased NAD+ levels as well as impairment of ATP production. Furthermore, $\mathrm{NAD}+$ affects neuronal health and survival through the regulation of the balance between mitochondrial biogenesis and mitophagy, which are controlled by the NAD+/SIRT1- PGC-1a pathway and the DAF16/FOXO3 pathway [84]. Decreased NAD+ levels can compromise mitophagy and trigger the accumulation of misfolded proteins leading to neuronal death [85]. Mitochondria play a part in the regulation of diverse physiological functions by providing energy for the majority of intracellular processes necessary for vital functions [86], and its optimum functioning is crucial for overall cell survival. Mitochondrial dysfunction decreases neuronal glucose which causes a decrease in Ach, ChAT, and ATP and increase in AchE. These lead to oxidative damage, DNA damage, and neuroinflammation after that it causes learning and memory impairment and neurotoxicity [87].

\section{CONCLUSION}

Accumulation of dysfunctional mitochondria and impairment in neuronal autophagy leading to impaired mitophagy are prominent features of AD. Although it remains to be determined if the presence of dysfunctional mitochondria is a consequence of inefficient mitophagy, or whether mitochondrial dysfunction is an early event preceding impairment of the autophagy/mitophagy systems, the concept of mitophagy and therapeutic viability of mitophagy is yet to be revealed as it holds immense potential.

\section{AUTHORS' CONTRIBUTIONS}

Author has reviewed the updated and recent developments of literature relevant to the subject and has incorporated it in the shape of the present review article.

\section{CONFLICTS OF INTEREST}

The authors have no conflicts of interest.

\section{REFERENCES}

1. Picard M, Wallace DC, Burelle Y. The rise of mitochondria in medicine. Mitochondrion 2016;30:105-16.

2. Nunnari J, Suomalainen A. Mitochondria: In sickness and in health. Cell 2012;148:1145-59.

3. Lane N, Martin W. The energetics of genome complexity. Nature 2010;467:929-34.

4. Martin WM. The origin of mitochondria. Nat Educ 2010;3:58

5. Lemasters JJ. Modulation of mitochondrial membrane permeability in pathogenesis, autophagy and control of metabolism. J Gastroenterol
Hepatol 2007;22 Suppl 1:S31-7.

6. Galluzzi L, Kepp O, Trojel Hansen C and Kroemer G. Mitochondrial control of cellular life, stress, and death. Circ Res 2012;111:1198-207.

7. Mitochondrial Medicine Society's Committee on Diagnosis, Haas RH, Parikh S, Falk MJ, Saneto RP, Wolf NI, et al. The in-depth evaluation of suspected mitochondrial disease. Mol Genet Metab 2008;94:16-37.

8. Wallace DC, Fan W. Energetics, epigenetics, mitochondrial genetics. Mitochondrion 2010;10:12-31.

9. Brand MD, Nicholls DG. Assessing mitochondrial dysfunction in cells. Biochem J 2011;435:297-312.

10. Sazanov LA. A giant molecular proton pump: Structure and mechanism of respiratory complex I. Nat Rev Mol Cell Biol 2015;16:375-88.

11. Legros F, Malka F, Frachon P, Lombe`s A, Rojo M. Organization and dynamics of human mitochondrial DNA. J Cell Sci 2004;117:2653-62.

12. Eichner LJ, Giguère V. Estrogen related receptors (ERRs): A new dawn in transcriptional control of mitochondrial gene networks. Mitochondrion 2011;11:544-52.

13. Schon EA, DiMauro S, Hirano M, Gilkerson RW. Therapeutic prospects for mitochondrial disease. Trends Mol Med 2010;16:268-76.

14. Davis RE, Williams M. Mitochondrial function and dysfunction: An update. J Pharmacol Exp Ther 2012;342:598-607.

15. Lugo-Huitrón R, Blanco-Ayala T, Ugalde-Muñiz P, Carrillo-Mora P, Pedraza-Chaverrí J, Silva-Adaya D, et al. On the antioxidant properties of kynurenic acid: Free radical scavenging activity and inhibition of oxidative stress. Neurotoxicol Teratol 2011;33:538-47.

16. Wallace DC. A mitochondrial paradigm of metabolic and degenerative diseases, aging, and cancer: A dawn for evolutionary medicine. Annu Rev Genet 2005;39:359-407.

17. Schapira AH. Mitochondrial disease. Lancet 2006;368:70-82.

18. Reddy $\mathrm{PH}$. Role of mitochondria in neurodegenerative diseases: Mitochondria as a therapeutic target in Alzheimer's disease. CNS Spectr 2009; 14:8-13.

19. Collier TJ, Kanaan NM, Kordower JH. Aging and Parkinson's disease: Different sides of the same coin? Mov Disord 2017;32:983-90.

20. Sheridan C, Martin SJ. Mitochondrial fission/fusion dynamics and apoptosis. Mitochondrion 2010;10:640-8.

21. Chan DC. Mitochondrial fusion and fission in mammals. Annu Rev Cell Dev Biol 2006;22:79-99.

22. Theurey P, Pizzo P. The aging mitochondria. Genes (Basel) 2018;9:175.

23. Pero RW, Roush GC, Markowitz MM, Miller DG. Oxidative stress, DNA repair, and cancer susceptibility. Cancer Detect Prev 1990;14:555-61.

24. O'Dell TJ, Hawkins RD, Kandel ER, Arancio O. Tests of the roles of two diffusible substances in long-term potentiation: Evidence for nitric oxide as a possible early retrograde messenger. Proc Natl Acad Sci U S A 1991;88:11285-9.

25. Knapp LT, Klann E. Role of reactive oxygen species in hippocampal long-term potentiation: Contributory or inhibitory? J Neurosci Res 2002; 70:1-7

26. Samina S. Oxidative stress and the central nervous system. J Pharm Exp Ther 2017;360:201-5.

27. Gross NJ, Getz GS, Rabinowitz M. Apparent turnover of mitochondrial deoxyribonucleic acid and mitochondrial phospholipids in the tissues of the rat. J Biol Chem 1969;244:1552-62.

28. Chomyn A, Attardi G. MtDNA mutations in aging and apoptosis. Biochem Biophys Res Commun 2003;304:519-29.

29. Trifunovic A, Wredenberg A, Falkenberg M, Spelbrink JN, Rovio AT, Bruder CE, et al. Premature ageing in mice expressing defective mitochondrial DNA polymerase. Nature 2004;429:417-23.

30. Kohen R, Beit-Yannai E, Berry EM, Tirosh O. Overall low molecular weight antioxidant activity of biological fluids and tissues by cyclic voltammetry. Methods Enzymol 1999;300:285-96.

31. Kohen R, Nyska A. Oxidation of biological systems: Oxidative stress phenomena, antioxidants, redox reactions, and methods for their quantification. Toxicol Pathol 2002;30:620-50.

32. Saso L, Firuzi O. Pharmacological applications of antioxidants: Lights and shadows. Curr Drug Targets 2014;15:1177-99.

33. Chance B, Schoener B, Oshino R, Itshak F, Nakase Y. Oxidationreduction ratio studies of mitochondria in freeze-trapped samples. $\mathrm{NADH}$ and flavoprotein fluorescence signals. J Biol Chem 1979; 254:4764-71.

34. Anderson G, Maes M. Oxidative/nitrosative stress and immunoinflammatory pathways in depression: Treatment implications. Curr Pharm Des 2014;20:3812-47.

35. $\mathrm{Gu} \mathrm{Y}$, Dee $\mathrm{CM}$, Shen J. Interaction of free radicals, matrix metalloproteinases and caveolin-1 impacts blood-brain barrier permeability. Front Biosci (Schol Ed) 2011;3:1216-31

36. Albrecht P, Lewerenz J, Dittmer S, Noack R, Maher P, Methner A, et al. 
Mechanisms of oxidative glutamate toxicity: The glutamate/cystine antiporter system xc- as a neuroprotective drug target. CNS Neurol Disord Drug Targets 2010;9:373-82.

37. Nguyen D, Alavi MV, Kim KY, Kang T, Scott RT, Noh YH, et al. A new vicious cycle involving glutamate excitotoxicity, oxidative stress and mitochondrial dynamics. Cell Death Dis 2011;2:e240.

38. Wang X, Michaelis EK. Selective neuronal vulnerability to oxidative stress in the brain. Front Aging Neurosci 2010;2:12

39. Solanki N, Alkadhi I, Atrooz F, Patki G, Salim S. Grape powder prevents cognitive, behavioral, and biochemical impairments in a rat model of posttraumatic stress disorder. Nutr Res 2015;35:65-75.

40. Masood A, Nadeem A, Mustafa SJ, O'Donnell JM. Reversal of oxidative stress-induced anxiety by inhibition of phosphodiesterase-2 in mice. J Pharm Exp Ther 2008;326:369-79

41. Popov VI, Bocharova LS. Hibernation-induced structural changes in synaptic contacts between mossy fibres and hippocampal pyramidal neurons. Neuroscience 1992;48:53-62.

42. Bearden CE, Thompson PM, Avedissian C, Klunder AD, Nicoletti M, Dierschke N, et al. Altered hippocampal morphology in unmedicated patients with major depressive illness. ASN Neuro 2009;1:e00020.

43. Uysal N, Tugyan K, Aksu I, Ozbal S, Ozdemir D, Dayi A, et al. Agerelated changes in apoptosis in rat hippocampus induced by oxidative stress. Biotech Histochem 2012;87:98-104.

44. Wong YC, Holzbaur EL. Autophagosome dynamics in neurodegeneration at a glance. J Cell Sci 2015;128:1259-67.

45. Fimia GM, Stoykova A, Romagnoli A, Giunta L, Di Bartolomeo S, Nardacci R, et al. Ambral regulates autophagy and development of the nervous system. Nature 2007:447:1121-5.

46. Komatsu M, Waguri S, Chiba T, Murata S, Iwata J, Tanida I, et al. Loss of autophagy in the central nervous system causes neurodegeneration in mice. Nature 2006;441:880-4.

47. Hara T, Nakamura K, Matsui M, Yamamoto A, Nakahara Y, SuzukiMigishima $\mathrm{R}$, et al. Suppression of basal autophagy in neural cells causes neurodegenerative disease in mice. Nature 2006;441:885-9.

48. Lipinski MM, Zheng B, Lu T, Yan Z, Py BF, Ng A, et al. Genomewide analysis reveals mechanisms modulating autophagy in normal brain aging and in Alzheimer's disease. Proc Natl Acad Sci U S A 2010; 107:14164-9.

49. Rubinsztein DC, Marin OG, Kroemer G. Autophagy and aging. Cell 2011; 146:682-95

50. Marta MV. Neuronal mitophagy in neurodegenerative diseases. Front Mol Neurosci 2017;10:64.

51. Thompson P. Mitochondrial quality control as potential therapy. Med Nous 2018;2018;8-9.

52. Sas K, Szabó E, Vécsei L. Mitochondria, oxidative stress and the kynurenine system, with a focus on ageing and neuroprotection. Molecules 2018;23:E191.

53. Kissova I, Deffieu M, Manon S, Camougrand N. Uth1p is involved in the autophagic degradation of mitochondria. J Biol Chem 2004; 279:39068-74.

54. Rodriguez-Enriquez S, Kim I, Currin RT, Lemasters JJ. Tracker dyes to probe mitochondrial autophagy (mitophagy) in rat hepatocytes. Autophagy 2006;2:39-46.

55. Wild P, McEwan DG, Dikic I. The LC3 interactome at a glance. J Cell Sci 2014;127:3-9.

56. McWilliams TG, Prescott AR, Allen GF, Tamjar J, Munson MJ, Thomson C, et al. Mito-QC illuminates mitophagy and mitochondrial architecture in vivo. J Cell Biol 2016;214:333-45.

57. Marzetti E, Csiszar A, Dutta D, Balagopal G, Calvani R, Leeuwenburg C, et al. Role of mitochondrial dysfunction and altered autophagy in cardiovascular aging and disease: From mechanisms to therapeutics. Am J Physiol Heart Circ Physiol 2013;305:H459-76.

58. Diot A, Hinks-Roberts A, Lodge T, Liao C, Dombi E, Morten K, et al. A novel quantitative assay of mitophagy: Combining high content fluorescence microscopy and mitochondrial DNA load to quantify mitophagy and identify novel pharmacological tools against pathogenic heteroplasmic mtDNA. Pharmacol Res 2015;100:24-35.

59. Strappazzon F, Nazio F, Corrado M, Cianfanelli V, Romagnoli A, Fimia GM, et al. AMBRA1 is able to induce mitophagy via LC3 binding, regardless of PARKIN and p62/SQSTM1. Cell Death Differ 2015; 22:419-32

60. Chen M, Chen Z, Wang Y, Tan Z, Zhu C, Li Y, et al. Mitophagy receptor FUNDC1 regulates mitochondrial dynamics and mitophagy. Autophagy 2016;12:689-702.

61. Gao F, Chen D, Si J, Hu Q, Qin Z, Fang M, et al. The mitochondrial protein BNIP3L is the substrate of PARK2 and mediates mitophagy in PINK1/PARK2 pathway. Hum Mol Genet 2015;24:2528-38.
62. Braak H, Braak E. Staging of Alzheimer's disease-related neurofibrillary changes. Neurobiol Aging 1995;16:271-8.

63. Price JL, Davis PB, Morris JC, White DL. The distribution of tangles, plaques and related immunohistochemical markers in healthy aging and Alzheimer's disease. Neurobiol Aging 1991;12:295-312.

64. Swerdlow RH, Khan SM. A mitochondrial cascade hypothesis for sporadic Alzheimer's disease. Med Hypotheses 2004;63:8-20.

65. Nixon RA, Yang DS, Lee JH. Neurodegenerative lysosomal disorders: A continuum from development to late age. Autophagy 2008;4:590-9.

66. Nixon RA. The role of autophagy in neurodegenerative disease. Nat Med 2013;19:983-97.

67. Ashrafi G, Schwarz TL. PINK1- and PARK2-mediated local mitophagy in distal neuronal axons. Autophagy 2015;11:187-9.

68. Mattson MP, Gleichmann M, Cheng A. Mitochondria in neuroplasticity and neurological disorders. Neuron 2008;60:748-66.

69. Cheng A, Wan R, Yang JL, Kamimura N, Son TG, Ouyang X, et al. Involvement of PGC-1 $\alpha$ in the formation and maintenance of neuronal dendritic spines. Nat Commun 2012;3:1250.

70. Mozdy AD, McCaffery JM, Shaw JM. Dnm1p GTPase-mediated mitochondrial fission is a multi-step process requiring the novel integral membrane component fis1p. J Cell Biol 2000;151:367-80.

71. Labrousse AM, Zappaterra MD, Rube DA, van der Bliek AM. C. Elegans dynamin-related protein DRP-1 controls severing of the mitochondrial outer membrane. Mol Cell 1999;4:815-26.

72. Duvezin-Caubet S, Koppen M, Wagener J, Zick M, Israel L, Bernacchia A, et al. OPA1 processing reconstituted in yeast depends on the subunit composition of the m-AAA protease in mitochondria. Mol Biol Cell 2007; 18:3582-90

73. Burté F, Carelli V, Chinnery PF, Yu-Wai-Man P. Disturbed mitochondrial dynamics and neurodegenerative disorders. Nat Rev Neurol 2015;11:11-24.

74. DuBoff B, Feany M, Götz J. Why size matters - balancing mitochondrial dynamics in Alzheimer's disease. Trends Neurosci 2013;36:325-35.

75. Cho DH, Nakamura T, Fang J, Cieplak P, Godzik A, Gu Z, et al. S-nitrosylation of drp1 mediates beta-amyloid-related mitochondrial fission and neuronal injury. Science 2009;324:102-5.

76. Rice AC, Keeney PM, Algarzae NK, Ladd AC, Thomas RR, Bennett JP Jr, et al. Mitochondrial DNA copy numbers in pyramidal neurons are decreased and mitochondrial biogenesis transcriptome signaling is disrupted in Alzheimer's disease hippocampi. J Alzheimers Dis 2014; 40:319-30

77. Kiriyama Y, Nochi H. The function of autophagy in neurodegenerative diseases. Int J Mol Sci 2015;16:26797-812.

78. Walsh DM, Klyubin I, Fadeeva JV, Cullen WK, Anwyl R, Wolfe MS, et al. Naturally secreted oligomers of amyloid beta protein potently inhibit hippocampal long-term potentiation in vivo. Nature 2002; 416:535-9.

79. Peric A, Annaert W. Early etiology of Alzheimer's disease: Tipping the balance toward autophagy or endosomal dysfunction? Acta Neuropathol 2015;129:363-81.

80. Gwon AR, Park JS, Arumugam TV, Kwon YK, Chan SL, Kim SH, et al. Oxidative lipid modification of nicastrin enhances amyloidogenic $\gamma$-secretase activity in Alzheimer's disease. Aging Cell 2012;11:559-68.

81. Gong CX, Grundke-Iqbal I, Iqbal K. Targeting tau protein in Alzheimer's disease. Drugs Aging 2010;27:351-65.

82. Reddy PH. Abnormal tau, mitochondrial dysfunction, impaired axonal transport of mitochondria, and synaptic deprivation in Alzheimer's disease. Brain Res 2011;1415:136-48.

83. Kondadi AK, Wang S, Montagner S, Kladt N, Korwitz A, Martinelli P, et al. Loss of the m-AAA protease subunit $\mathrm{AFG}_{3} \mathrm{~L}_{2}$ causes mitochondrial transport defects and tau hyperphosphorylation. EMBO J 2014;33:101126.

84. Fang EF, Scheibye-Knudsen M, Brace LE, Kassahun H, SenGupta T, Nilsen $\mathrm{H}$, et al. Defective mitophagy in XPA via PARP-1 hyperactivation and NAD $(+) /$ SIRT1 reduction. Cell 2014;157:882-96.

85. Zhou M, Ottenberg G, Sferrazza GF, Hubbs C, Fallahi M, Rumbaugh G, et al. Neuronal death induced by misfolded prion protein is due to $\mathrm{NAD}+$ depletion and can be relieved in vitro and in vivo by NAD+ replenishment. Brain 2015;138:992-1008.

86. Sasikala S, Lakshminarasaiah S, Malepati DN. Effect of Centella asiatica (Linn) leaves on selective mitochondrial and cytosolic enzymes in streptozotocin induced diabetic rats. Int J Pharm Pharm Sci 2015; 7:322-5.

87. Deka P, Kumar A. Pharmacological evaluation of triphala churna in streptozotocin (I. C. V.) Induced dementia in rats. Int J Pharm Pharm Sci 2018;10:97-105. 